\title{
We need answers
}

To the Editor - Within the European Seventh Framework (FP7) Programme, there are 34 projects dedicated to nanomaterial toxicology worth over $€ 100$ million $^{1}$. This investment was necessary to establish public confidence, but nanotoxicology studies have not yet provided any clear and unequivocal answers on the toxicity of nanomaterials, as was highlighted in your recent Editorial ${ }^{2}$.

At present, nanomaterial toxicity is assessed by following test protocols developed by the International Organization for Standardization (ISO) and/or the Organisation for Economic Co-operation and Development (OECD) $)^{3,4}$. These guidance documents were established based on results of early investigations into the toxicity of nanomaterials, where size, shape, chemistry, homogeneity and processing history of the nanomaterial were shown to be important. Consequently, the need to provide physicochemical data to support toxicological findings is increasingly important. Although current measurement guidelines will inform researchers as to which physicochemical characteristics should be measured, the quality of the data generated is being questioned. In particular, current techniques may not be suitable for all nanomaterial samples (for example, if the sample has a polydisperse shape and/or size, or if it is in a complex medium). Furthermore, imaging techniques such as transmission electron microscopy may give results that are either statistically not significant or highly operator dependent. The need to develop new and better measurement techniques is clear but it will take time and substantial research.

If our immediate goal is to effectively regulate without hampering public perception on the benefits of nano-related products, first, all stakeholders should agree on the definition of a nanomaterial, and the measurement methods that would support this definition. Second, the phases of the nanomaterial life cycle should be identified so the risk associated with each phase (from synthesis to product) can be assessed. For example, carbon nanotubes may be considered high risk in a factory setting where aerosol exposure can be problematic, but may be rendered medium or low risk when encapsulated in a composite matrix. Finally, simple test methods are needed to identify these risk categories. Instead of attempting to report a number of physicochemical properties at every phase of the nanomaterial life-cycle, we need to consider if there are some overarching parameters that can easily and rapidly categorize the risk of toxicity.

References

1. European Commission European Nanosafety Cluster Compendium (2012); http://go.nature.com/xLp2QR 2. Nature Nanotech. 7, 545 (2012).

3. International Organization for Standardization ISO/TR 13014:2012: Nanotechnologies - Guidance on Physico-chemical Characterization of Engineered Nanoscale Materials for Toxicologic Assessment (2012); http://www.iso.org/iso/home.htm

4. Organisation for Economic Co-operation and Development List of Manufactured Nanomaterials and List of Endpoints for Phase One of the Testing Programme (2008); http://go.nature.com/aJBhhe

\section{Acknowledgements}

The authors gratefully acknowledge Steffi Friedrich from the Nanotechnology Industries Association, Richard Brown and John Jarman for their contributions to the work.

\section{Disclaimer}

The views expressed in this paper are those of the authors and do not necessarily represent those of the National Physical Laboratory or NPL Management Limited.

\section{Ratna Tantra* and Alex Shard}

National Physical Laboratory, Hampton Road, Teddington, Middlesex TW11 OLW, UK.

*e-mail: ratna.tantra@npl.co.uk

\section{Broaden the discussion}

To the Editor - We agree with your Editorial 'Join the dialogue' (Nature Nanotech. 7, 545; 2012) that there is a need for guidelines on materials characterization requirements when reporting

nanotoxicology research. However, defining a minimum set of requirements will not substitute for a rigorous peer-review process because materials characterization per se does not necessarily mean that measurements have been performed using state-of-the-art methods, or that the parameters are directly associated with the observed biological effects. Different types of information (and consequently, the use of different methods) are needed depending on the purpose of the study. Materials characterization in the relevant biological matrix is sorely needed and developing methods to do this is important. The use of reference materials can facilitate comparisons between nanotoxicological studies, and such materials should be widely available to the community, for example, through a central repository.
In addition to pinpointing hazard mechanisms and/or ranking nanomaterials as a function of their hazard using in vitro and/or in vivo models, we think it is necessary to broaden the discussion to consider the types of information needed to enhance the quality and comparability of exposureassessment studies (Brouwer, D. et al. Ann. Occup. Hyg. 56, 1-9; 2012). Nanomaterial risk is a function of hazard and exposure, and at the heart of both disciplines is the need to understand the properties of the nanomaterials in question. We fully agree with Schrurs and Lison (Nature Nanotech. 7, $546-548 ; 2012)$ that more coherence is needed in the way that nanotoxicological studies are performed, but this also applies to nanosafety research in a broader sense. One of the main objectives of the European NanoSafety Cluster (www.nanosafetycluster.eu) is to improve coherence in the area of nanosafety research and to harmonize studies in this field. Furthermore, the Cluster aims to avoid duplicating work, and to provide a forum for discussion and bottom-up planning of research activities in the European Union. To this end, the Cluster (of the Sixth Framework Programme (FP6) and FP7 projects along with key national projects) is organized into several working groups focusing on: materials, hazard, exposure, risk, (mathematical) modelling, databases and dissemination. The Cluster is currently putting together a "chart of state-of-the-art methodologies in nanosafety research", and the issue of whether a set of minimal requirements for materials characterization can be defined has been taken on board.

Acknowledgements

With contributions from members of the European NanoSafety Cluster: Derk Brouwer, Kenneth Dawson, Andrea Haase, Thomas Kuhlbusch and Ulla Vogel.

Bengt Fadeel ${ }^{1 \star}$ and Kai Savolainen ${ }^{2}$ ${ }^{1}$ Institute of Environmental Medicine, Karolinska Institutet, Stockholm SE-171 77, Sweden, ${ }^{2}$ Finnish Institute of Occupational Health, Helsinki FI-00250, Finland.

*e-mail: bengt.fadeel@ki.se 